\title{
Building a (Virtual) Aleph: The Visual Transformation of a Tiny Cosmogony
}

\author{
Elif Ayiter \\ Sabanci University, Istanbul, Turkey
}

\section{ABSTRACT}

This article discusses the transformation of a tiny three dimensional artwork created in the metaverse which takes its inspiration from Jorge Luis Borges's story of 'the Aleph' - a tale that revolves around a minute artifact which is "one of the points in space that contains all other points." (Borges 1945)

The visualization of a concept as complex as 'the Aleph' led the author of the project through a process which started from Cartesian precepts, from where it transformed itself into an investigation of 'code' as a basal, life-giving presence that is abstract, enigmatic, and hermetic in its essence. This correlation between 'code' and the magical nature of 'the Aleph' has led to further considerations regarding the significance of such a marvelous object within virtual worlds. Accordingly, this text will conclude with the description of an intriguing, novel usage for 'the Aleph' that materializes as a Time Machine for virtual world avatars.

Keywords: 3D; Art; the Aleph; Avatar; Jorge Luis Borges, Metaverse; Process; Time Machine; Virtual Worlds; Visualization.

\section{THE ALEPH AS CYBER ARTIFACT}

The project under discussion in this text revolves around the transformation process of a miniature, volumetric artwork created in the metaverse - a virtual construct that is so small that it easily fits onto the fingertip of an avatar. As such, the object is one that can only be scrutinized by using a device that stands in lieu of a microscope, in this instance the metaverse avatar's eyes, which can be used to view the object in 'mouselook.'

Consequently, what is under discussion here is an object, the usage of which relies entirely upon the eyes of a virtual agent: Only through the agency of our avatar can we see and interact with 'the Aleph' that I have built in the metaverse of the OpenSim. It is, in other words, a virtual object for virtual beings that are extensions of ourselves.

The work takes its inspiration from Jorge Luis Borges's story of 'the Aleph' which revolves around a wondrous artifact, "a small iridescent sphere of almost unbearable brilliance” that enables anyone who gazes into it (to) see everything in the universe from every angle simultaneously, without distortion, overlapping or confusion, "the only place on earth where all places are seen from every angle, each standing clear, without any confusion or blending.” (Borges 1945) 
Although the story of 'the Aleph' was written at a time well before developments in cyber technologies had reached a level of fruition to let us claim that Borges may have been inspired by computer technologies when he wrote his tale; nevertheless 'The Aleph' has compelled many writers and theoreticians of cyber cultures to draw inspirations from, and point at analogies and similarities between the tiny shining sphere of Borges's imagination and developments in cyberworlds that have transpired over the past few decades.

\section{Cyber Beings, Posthumanism and the Borgesian Flux}

Ralph Dumain notes that 'the Aleph' replicates many of the themes found in Borges's stories, such as the one concerning infinity and infinite divisibility; the paradox of the reduplication of the universe in a microcosm which results in cosmic destabilization as well as an anxiety of infinite regress; some liminal talismanic object of perfection representing the aforementioned destabilization (such as the Aleph); the impossibility of living with the consciousness of total flux; the instability underlying the illusion of permanence. Slippage of identity and memory (seen as illusory abstractions), especially manifesting through a dissolution of language and persona into extreme nominalism; chance encounters and the sense of breaking a taboo by encountering structures such as the Aleph; as well as shrinking back from such limit states to the ordinariness of the finite world are amongst the recurrent motifs of these texts. (Dumain 2010)

This listing of Borgesian themes and motifs seems to dovetail remarkably well with the enigmatic nature of virtual beings and constructs whose existence comes about through bits and not through atoms. This change of basal building material results in an environment in which virtual presences (objects as well as personas such as avatars) can infinitely be manipulated with no discernible loss, through processes in which even concrete objects can be created through abstract notation; can be copied, duplicated and re-combined to generate perpetually novel artifacts; varying 'levels of detail' which become apparent when zoomed in and out; resulting in conditions in which concepts such as scale, fixedness, stability and uniqueness, become irrelevancies.

This enigmatic, fluid state resides in the microstructure of the computational medium: Processes that move physical atoms around constitute the irreversible aspects of traditional work, in which most operations are beyond recall. By contrast, the microstructure of the digital medium is comprised of bits which are specified arrangements of symbols. Accordingly, in the microstructure of the computational medium, arrangements and values can always be reconstructed, their previous states can be stored and recalled, additional instances and versions can be replicated, resulting in a continuously workable medium. (McCullough 1996: 213-214)

Alan Kay defines this state as follows:

"The protean nature of the computer is such that it can act like a machine or like a language to be shaped and exploited. It is a medium that can dynamically simulate the details of any other medium, including media that cannot exist physically. It is not a tool, although it can act like many tools. It is the first metamedium, and as such it has degrees of freedom for representation and expression never before encountered and as yet barely investigated." (Kay 1984: 59) 
I am certainly not alone in my fascination with 'the Aleph' as a talismanic object of perfection that affects cyber states of being. I rest my query on a large body of Cyberpunk literature in which Borgesian themes and motifs, and especially 'the Aleph,' have been a central concern and a source of inspiration:

"Personal cosmologies, or the development of technological microcosms of the larger world, have been a major theme in cyberpunk... [and] ...'the Aleph,' as a symbol of united infinity- a single glance within which all visible reality is manifest simultaneously, is extensively covered in cyberpunk literature in which the question as to whether technology can reproduce Borges's idea of the aleph, 'the microcosm of the alchemists and Cabbalists, our true proverbial friend, the multum in parvo' is often asked.

That is only a part of the question this research needs to ask, as the real question for cyberpunk is why would someone desire a microcosm, an aleph?” (McCune 2009)

A compelling answer to this question may come from William Gibson who writes about an Aleph which is a biochip that can store vast amounts of data, a neuralgic centre in consciousness, or a self-terminal where our most intimate memories are projected. Thus one of his protagonists enters the novel 'Mona Lisa Overdrive' in a highly unusual state - his body is in a medical coma of sorts, and a black box is attached to his head. The box is later revealed to be something called an 'Aleph' which essentially exists as a form of AI, a whole virtual universe wired directly into the hero's nervous system. While his body lies in an inert state, his consciousness roams freely throughout the Aleph which is a virtual recreation of the whole universe.

However, Gibson is not the only cyberpunk author who is fascinated by Borgesian concepts. In their 2009 book chapter Stefan Herbrechter and Ivan Callus state that much of this fascination has to do with the way in which many of Borges's writings can be read as unsurpassable experiments in how to make thinkable the impossible and the unrepresentable. According to Herbrechter and Callus obsession with the posthuman, as that which reconfigures the actual and the possible once technology re-engineers human potential, is overwhelmingly present throughout Borges's oeuvre. (Herbrechter and Callus 2007: 180) The authors set up a special correlation between Borges and post-humanist theorist Katherine Hayles, who in her book 'How We Became Posthuman' named Borges as one of the precursors to the cybernetic notion of reflexivity, "the system generating a reality [that] is shown to be part of the reality it makes." (Hayles 1999: 8)

Hayles examines Borges, and in particular 'The Aleph,' in relation to the mathematical notion of 'field concept,' on the basis of interconnectivity and self-referentiality. Borges, according to Hayles, is attracted to the field concept, "because its discontinuities reveal that everything, including itself, is no more than a game" and this leads "to the discovery of paradoxes of self-referentiality," indefinite expansion and the possibility for creating "new kinds of Strange Loops” in his writings. "Where Borges's fiction differs from scientific models of the field concept, however, is in using the concept to suggest that everything, including reality, is a fiction.” Borges, Hayles says, uses these paradoxes to exploit rather than to suppress any inconsistencies, "because he hopes to use them to reveal the essential fictionality of the model. His intent is thus subversive.” (Hayles 1984: 138)

Returning to Herbrechter and Callus, we come across the notion that Borges's 'Aleph' might be more powerful in its self-referentiality than Hayles states. Although there is a circular structure that seems to try to recapture an infinite series, it nevertheless never manages to do so with a 'twist' - a 'Strange Loop' 
rather in the manner of a Moebius strip, which does more than just turn itself into a simple cybernetic feedback structure. (Herbrechter and Callus 2007: 189)

\section{BUILDING A VIRTUAL ALEPH}

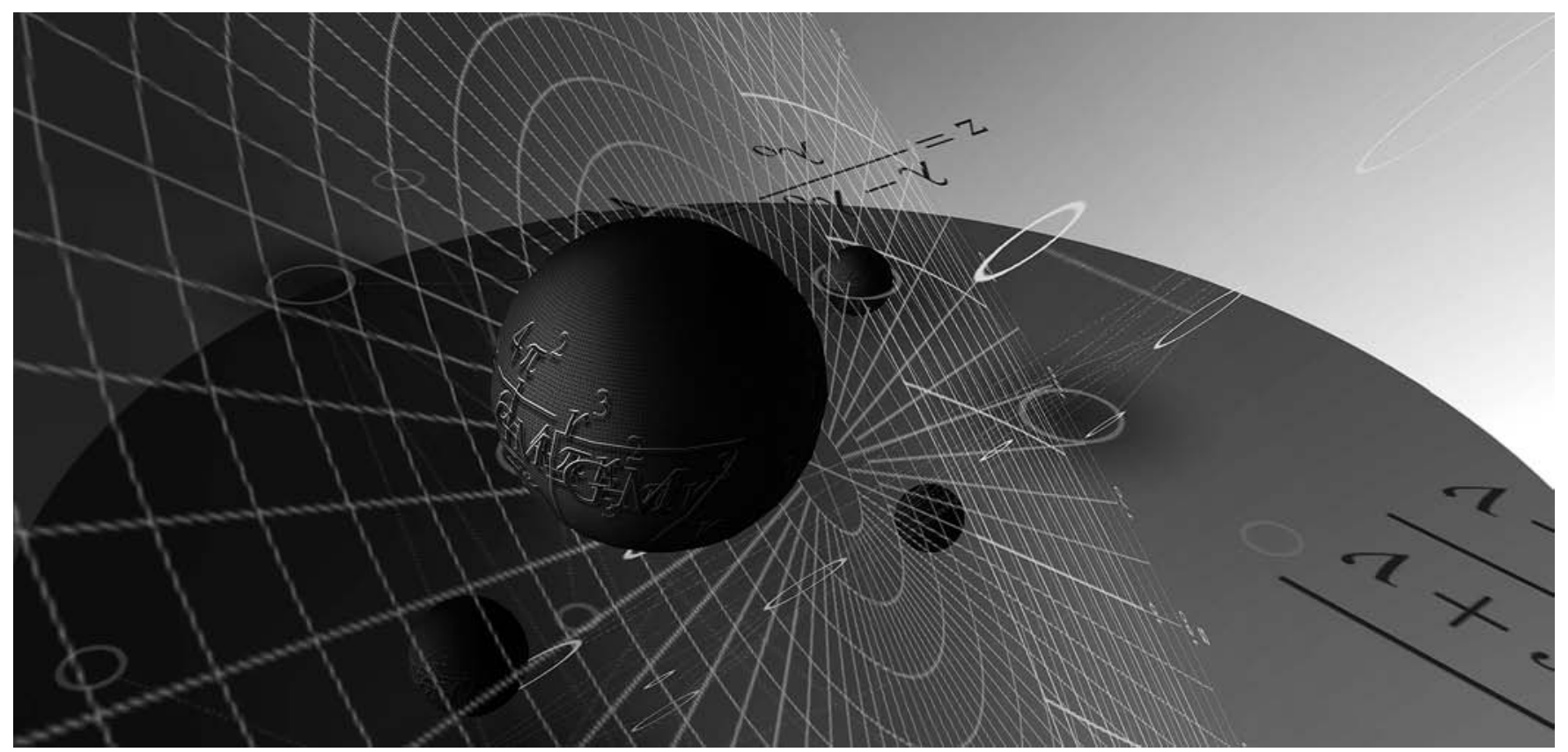

Figure 1. 'Azimuth.' (Elif Ayiter, aka. Alpha Auer, Second Life, 2013.

My personal journey with 'the Aleph' started out as a tiny solar system that was created in a virtual world (Figure 1). The idea was to turn something that we automatically tend to think of as infinitely large into something that is very small, and thereby establish the contradiction that lies at the heart of 'the Aleph.' The inspirations for building something very small that would hold something very large, however, came from physical, real-life, historic sources such as Chinese ivory miniatures and the entire Koran written on a grain of rice.

This tiny architecture was named 'Azimuth,' in accordance with a number of Cartesian typographic elements that I deconstructed to build a decentralized planetary system that nevertheless revolved around a centralized solar chart. Although the solar system was meant to be contradictory and confusing when considered in a Cartesian sense, I also wanted reminders of Cartesian signifiers, to which end I used astronomical formulas that were wrapped around spheres that were meant to represent the planets of this idiosyncratic solar system. These spheres moved randomly around a tilted diagram that showed the altitude and azimuth of the Sun for a range of local solar times from sunrise to sunset at specific latitude. My many suns could not possibly be measured by such means, of course. And yet, there they would be bounded by, and indeed held ransom to the inflexible, logical, centralized beauty of this diagram.

However, as my building progressed, I soon began to realize that this strategy was far too literal, that it failed to address what was at the core of 'the Aleph,' as I had now begun to understand it through an extended journey of reading that took its trajectory from the story itself and that led me to places such as cyberpunk literature, the Kabala, numerology and its relation to the concept of 'code' as a basal life force 
that stretched from intangible abstractions such as mysticism and music to the tangibly concrete such as biology and the workings of computers. This reading material, as well as a fundamental dissatisfaction with what I was in the process of building, began to lead me away from an attempt at re-creating Borges's complex description of the wondrous object that allowed him to see "millions of acts both delightful and awful; not one of them occupied the same point in space, without overlapping or transparency" (Borges 1945) as something as immediately identifiable and as obvious as a solar system.

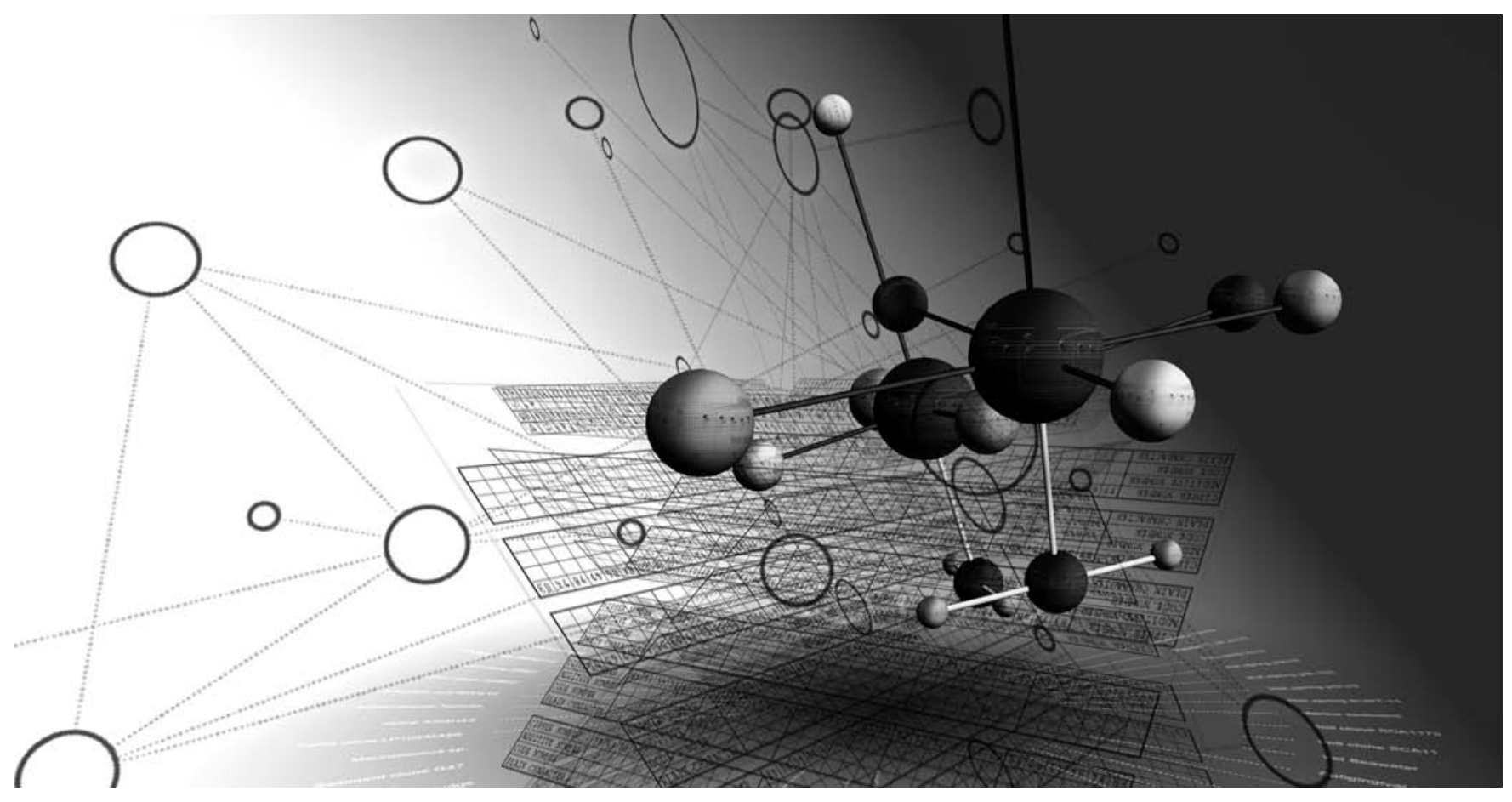

Figure 2. 'Cypher' Elif Ayiter, aka. Alpha Auer, Second life, 2013

A grasp of 'the Aleph' now seemed to me to be best possible through an acknowledgement of it as an 'Enigma,' as a presence that defies definition and description - indeed a non-presence or a non-entity that may be grasped through a visual conglomeration of 'code' as an identifier of enigma. This resulted in a novel stage of my visual journey with ‘the Aleph’: The project began to take shape as an evolving, three dimensional link-and-node structure built out of many different (and seemingly unrelated) visualizations of code.

At this stage I re-named the construct 'Cypher,' (Figure 2) a name that took its inspiration from the Renaissance school of the Cisternian Monks who appeared to have developed an occult system based upon Pythagorean Numerology and the philosophy of Agrippa that revolved around 'Cipher.' Yet another reason for giving this name to what I was about to build resided in my fascination with the double meaning of the term, since 'cipher' also means 'zero,' which as the smallest transfinite cardinal number is also called 'aleph-zero.'

Taking my investigation of the word 'Cipher' even further I found that in cryptography, a cipher denotes an algorithm for performing encryption or decryption, consisting of a series of well-defined steps that can be followed as a procedure. Thus, the next step in my travels that helped shape my second rendition of 
'the Aleph' brought me to Alan Turing who is so said to have said that "you could use, in effect, a theorem in logic which sounds to the untrained ear rather absurd; namely that from a contradiction, you can deduce everything” (Moon 2014: 7) - a statement which resounded strongly with what my idea of 'the Aleph' was, of how it should be visualized.

Consequently, the central body of 'Cypher' was constructed out of scans of Turing's Enigma codes which I placed upon a further body of code, in this case a phylogenetic tree diagram that showed the inferred evolutionary relationships among various biological species based upon similarities and differences in their physical and/or genetic characteristics. The third visual element that I brought into this conglomeration of code were the scores of Elgar's Enigma variations about which their author said:

"The Enigma I will not explain - its 'dark saying' must be left unguessed, and I warn you that the connection between the Variations and the Theme is often of the slightest texture; further, through and over the whole set another and larger theme 'goes', but is not played.... So the principal Theme never appears, even as in some late dramas ... the chief character is never on the stage." (Atkins 1934: 328330)

Just as the quote attributed to Turing above greatly inspired me, this quote of Elgar's also became highly relevant to my transformation of 'the Aleph' from 'Azimuth' to 'Cypher,' since in the latter project 'the chief character is never on stage,' while in its forerunner the concept of the solar system as a symbol of something as mysterious and closed as 'the Aleph' is glaringly apparent and manifest: Code runs through everything - from biological beings to electronic entities to mystical systems related to numerology - and yet, at its most potent, code is non-apparent, lives as the silent life-force in the background of the beings and systems that it animates.

\section{THE ALEPH AND THE AVATAR}

While the fascination that Borges's 'Aleph' holds for cyberpunk writers and new media theoreticians already justifies it being considered as a fully fledged virtual object in its own right, I hope to have added a modest twist to the discussion by building an 'Aleph' that is meant not for the scrutiny of human beings, as is the case in Borges's story, but instead this is a virtual artifact that exists in a virtual world and is presented to the scrutiny of virtual beings - only through the eyes of our avatar can we see 'the Aleph.'

As said at the onset of this text, the object is very small, easily fits on the fingertip of a virtual world avatar who emulates the size and proportions of a physical human being, cannot even be seen with the naked avatar eye, and requires devices such as metaverse viewer's camera to zoom in on it. And even then, the viewing of it is a precarious undertaking since at such infinitely close range the camera tends to shake and a clear view from a viewpoint that is external to the avatar can only be obtained by taking virtual screenshots of the object that freeze the screen while the shot is being taken to allow for a focused viewpoint. There is however another way of looking at the virtual 'Aleph' and that is through the eyes of the avatar, by going into what in virtual worlds is called 'the mouselook.'

A further question is connected to the question of scale that makes the viewing of the virtual 'Aleph' utterly reliant upon the eyes of the avatar: Given that in a virtual world what is small and what is big are rather relative conceptions, how would such a fluidity of scale affect the concept of 'the Aleph' as a tiny 
object that is yet big enough to be "the only place on earth where all places are seen from every angle, each standing clear, without any confusion or blending?"

How do we know how big the avatar is in relation to this wondrous miniscule object? Yes, virtual worlds give us measurements of 'virtual meters' - but what do these virtual meters mean exactly? In a metaverse not all avatars affect physical human sizes, instead oftentimes they can be extremely small, can even be invisible; or conversely they can be extremely large. Furthermore, body sizes are fluid and unfixed - the same avatar can be extremely small and extremely large almost simultaneously, or at such short intervals of change that it is almost a concurrent event.

Moreover, there seems to me to be a fascinating further relationship between 'the Aleph' and the avatar which I am presuming to connect to the Jewish myth of the Golem (a piece of clay that is given life through Kabalistic rituals by the writing of a name, the first letter of which is the Hebrew letter 'aleph') on its forehead: Just like the Golem, virtual world avatars come into life through the initial creation of a name. True, an avatar's name need not have the letter aleph as an initial in order for the virtual creature to come into being, but nevertheless the connections between the two naming processes that give life to a piece of clay and to a coded persona appears to me to be one to ponder upon further in future research and writings.

The relationship between the human behind the keyboard is riddled with complex ties that have been discussed at length by many researchers of the field, including Richard Bartle, for whom the final identification stage between handler and avatar is a fully fledged 'persona' stage in which the two entities have become seamlessly integrated to one another, to the point where an emotional separation is deemed to be inconceivable by the human behind the keyboard. (Bartle 2004: 156-165)

This powerful state of identification through virtual embodiment is noted upon by Benjamin Joerissen, who points at the correlation between the ideological affinity of the avatar and the human body: Drawing upon Plato, as well as the Sanskrit meaning of the word avatar itself, Joerissen says that within these doctrines the human body itself can be identified as the disparaged, earthbound hybrid carrier / avatar of higher, divine, non-physical attributes.

In a play upon Marshall McLuhan's famous statement "the medium is the message," Joerissen asks if soul is indeed 'form,' is then the body not the medium within which form becomes corporeal and as such the body becomes the very message that it carries. Furthermore, a recent, post-Cartesian shift in the attributes with which an avatar is endowed is also noteworthy: In the post-humanistic world of artist Stelarc the avatar is no longer the belittled, lesser manifestation of the higher 'form' but rather the 'upload' of a perishable, mortal physicality into the mundus possibilis of a virtual, non-corporeal space; an agent in the realization of a 'cybernetic platonic' state wherein technology may overcome the shackles of mortality (Joerissen 2008: 277-295).

Joerissen quotes Mark Hansen who points at a deep reaching biological/corporeal moment embedded within the virtual experience: Whilst placing the digital experience itself within the sensory organs of the biological body, Hansen ascribes a third element to digital embodiment, speaking of a "body submitted to and constituted by an unavoidable and empowering technical deterritorialization," (Hansen 2006: 20) a "body-in-code," that can only be realized in association with technology, and that, in its turn, can lead to unexpected self-perceptions in the human handler. Indeed Hansen endows this 
novel constellation with the capability of increasing the field of influence of the human operator as an embodied being. Thus, Hansen predicts a re-definition of the potential of the biological body through virtual embodiment. (Joerissen 2008: 277-295)

Writing several years before Mark Hansen, Frank Biocca points in the same direction when he notes upon a potential increase in the availability of kinesthetic and somatic interfaces which can be expected to vastly augment avatar agency into states of online hyperpresence:

“... it may be possible to develop a medium in which one feels greater 'access to the intelligence, intentions, and sensory impressions of another' than is possible in the most intimate face-to-face communication. Of course, it is hard for us now to imagine a medium that can create greater intimacy than face-to-face communication. But this misses the point of social presence and the very artifice of the body itself. ... for example, inner states might be communicated more vividly through the use of sensors that can amplify subtle physiological or nonverbal cues. These can augment the intentional and unintentional cues used in interpersonal communication to assess the emotional states and intentions of others." (Biocca 1999: 113-144)

Thus, the virtual world avatar is a compelling being that already deserves close scrutiny in light of our experiences today. Looking into the future, however, avatars appear to hold much promise for vastly enriched interchanges, not only between separate individuals but also in terms of reaching deeper understandings of our own selves - states which may well culminate into a broadening of human potential and creativity.

\section{CONCLUSION: TIME MACHINES, THE ALEPH AND THE AVATAR}

The potential of avatars as agents of deeper understandings of ourselves as well as the carriers of enriched interchanges between individuals has been explicated upon in the literature review above. Added to this should also be the potential that the coded nature of virtual worlds brings to a discussion that connects avatars to 'the Aleph.'

In their article entitled 'Leave your trace - the virtual time machine,' Selavy Oh and Stefan Glasauer propose a time machine that allows visiting the past which can be realized in a virtual world wherein digital data can be recorded and stored continuously, for revisiting at will. Since virtual worlds need not adhere to the laws of physics as we know them, time travel could happen in continuous time as in $\mathrm{H}$. G. Wells' famed novel, or it could be discontinuous with the possibility to jump from one point in time to another. (Oh and Glasauer 2014: 195-206)

Oh and Glasauer's notion is based upon the widely based practice of backing up digital content. For such a backup to function like a time machine however, backup has to happen not just every hour, but continuously, i.e., with the frame rate of the update of the virtual world. Once a quasi-continuous memory trace of all events in the world, including the actions of the avatars present at any given moment, is available, it is then also possible to playback this memory trace and such a playback, being the exact replicate of the former state of our virtual world, can now be visited at will.

However, even in a virtual world with all its possibilities some of the usual time-travel problems still would exist: the visitor from the future would be in a recorded scene, and not in a presence. Thus, the 
visitor would have to feel like a ghost: unable to interact with the world around her. Despite this missing agency, it would be possible to watch the visitor's own past appearance or visit any desired place, even if the visitor has not been there at that time. Of course, the time machine would not have to run in real time, but could allow fast-backward, watching the past in slow motion, or even stopping time to contemplate that one moment in all its aspects. (2014: 198)

The authors continue to ponder several more fascinating questions such as recursive states of being in time, for which one would just have to record what one did in a given moment in time and nothing more, since that moment in time itself has already been recorded: "You can visit the past in which you visited the past and see your former self visiting an even older self." (2014: 198)

In their study Oh and Glasauer's demonstrate that the Time Machine is an apparatus that is more than a potentiality or a mere figment of their imagination; that it is in fact a system that can operate now, today, on any metaverse server provided that there is enough storage space for the accumulated digital data needed to undertake such virtual journeys in time.

What is compelling for the purposes of this paper should of course be obvious: It is the remarkable extent the workings of a virtual Time Machine seem to correspond to Borges' description of 'the Aleph' as a thing that allows its viewer to witness "millions of acts both delightful and awful; not one of them occupied the same point in space, without overlapping or transparency.” (Borges 1945)

Could 'the Aleph' therefore be giving us a clue as to how the interface for a virtual Time Machine should be developed? In Borges's tale, great emphasis is placed upon the simultaneity of the viewing experience that 'the Aleph' provides, which he finds hard to translate into language. Indeed, Borges especially remarks upon this by saying "what my eyes beheld was simultaneous, but what I shall now write down will be successive, because language is successive." (Borges 1945) Borges was of course bound by the restrictions of a physical body which need not necessarily apply to the capabilities of virtual beings such as avatars. What may therefore need to be considered is not only what the interface of a virtual 'Aleph' as Time Machine would have to be like; but also how the receptor of its bounty - the avatar - may need to reach a novel state of evolution that is capable of processing and making sense of the massive influx of simultaneous data that it is confronted with.

Indeed, the evolution of the avatar - not only in terms of data processing capabilities but in a much broader sense as well - are further fascinating questions that are begging to be asked: What would happen if these virtual creatures (that seem to already have become our integral extensions in a very fundamental manner) were to also acquire autonomous behaviors of their own? What if our avatars continued to operate and, even more importantly, to observe and to emote in the virtual world in our absence? Although we are, of course, yet a ways removed from this state of posthuman being, there are already significant investigations by virtual artists and scientists regarding the creation of autonomous or semiavatars, most recently reported upon by Jeremy Turner et al who expound upon the challenges related to the creation of a semi-autonomous, 'self-absorbed' avatar called Qiezli in Second Life. (Turner et al 2015: 55-75)

In conclusion, with all this in mind, I cannot help but wonder what the encounter between such an autonomous or semi-autonomous avatar and a miraculously coded virtual 'Aleph' (that enables any avatar who gazes into it to see everything in the universe from every angle simultaneously, without 
distortion, overlapping or confusion) be like? What would our virtual doppelganger see and how would such exchange between 'the Aleph' and our virtual counterparts be transmitted back to us? Would we then finally be able to gaze into every hidden corner of the (virtual) universe through the coded eyes of our avatar?

\section{REFERENCES}

Atkins, I., (1934). Elgar's' Enigma'Variations, The Musical Times, 75(1094), 328-330.

Bartle, R., (2004). Designing Virtual Worlds, New Jersey, New Riders Publishing/Pearson, 156-165.

Biocca, F., (1999). The Cyborg's Dilemma: Progressive embodiment in virtual environments, Human Factors in Information Technology, vol 13, pp: 113-144.

Borges, J. L., (1945). The Aleph, trans. Norman Thomas di Giovanni, Retrieved April 12th 2015, http://www.phinnweb.org/links/literature/borges/aleph.html

Dumain, R., (2010). On 'The Aleph’ by Jorge Luis Borges: Observations and Questions, Retrived April 15th 2015, http://www.autodidactproject.org/my/borges_10_aleph.html

Hansen, M., (2006). Bodies in Code: Interfaces with Digital Media. Routledge, New York. p: 20.

Hayles, K. N., (1984). The Cosmic Web: Scientific Field Models and Literary Strategies in the Twentieth Century, Ithaca, NY: Cornell University Press. pp. 138, 142-143, 151

Hayles, K. N., (1999). How we Became Posthuman, Virtual Bodies in Cybernetics, Literature, and Informatics, Chicago, IL: University of Chicago Press, 8.

Herbrechter, S., Callus, I. (2007). Posthumanism in the Work of Jorge Luis Borges, in Latin American Cyberculture and Cyberliterature, ed. Claire Taylor and Thea Pitman, (p180), Liverpool, England, University of Liverpool Press.

Joerissen, B. (2008)., The Body is the Message. Avatare als visuelle Artikulationen, soziale Aktanten und hybride Akteure, Paragrana, 17(1). 277-295.

Kay, A. (1984)., Computer Software, Scientific American, 251(09), p. 59.

McCullough, M., (1996). Abstracting Craft: The Practiced Digital Hand, Boston, MIT Press, 213- 214.

McCune, Z. (2009)., United Infinity: Borges \& The Aleph, Retrieved April 2nd 2015.

http://thames2thayer.com/cyberpunk/?p=69, 
Moon, A., (2014)., Alan Turing and the Enigma Machine, e-book, Lulu.com, 7.

Oh, S., Glasauer, S., (2014). Leave your trace - the virtual time machine, Metaverse Creativity, 4(2), 195-206.

Turner, J., Pasquier, P., Nixon, M., (2015). Qiezli - A “Self-Absorbed” Creative Virtual Agent in Second Life, Metaverse Creativity, Intellect, 55-75. 